\title{
Argumentation Based Negotiation in Cognitive Radio Networks
}

\author{
Brent Horine, Ladislau Bölöni, and Damla Turgut \\ School of Electrical Engineering and Computer Science \\ University of Central Florida \\ Orlando, FL 32816-2362 \\ \{bhorine,lboloni,turgut\}@eecs.ucf.edu
}

\begin{abstract}
Cognitive radio networks allow for a more dynamic allocation of network resources (such as the radio spectrum) compared to conventionally engineered networks. We consider a scenario where each node acts as an autonomous agent, maintaining a knowledge base of the network conditions and pursuing its own communication goals. The agents use negotiation to reach agreements about the allocation of network resources and the configuration of forwarding paths.

The contribution of this paper is a novel, argumentation based negotiation framework. Instead of a simple exchange of offers, the agents also offer arguments in favor of their proposals and critiques of received offers. Arguments can contain relevant new information about the state of the network, allowing the agents to update their knowledge base. The system is governed by a collection of rules and policies, which can be invoked in the support of requests or, in some cases, to justify their denial.

To illustrate the proposed scheme, we designed a negotiation protocol and argumentation language for a communication model involving discrete service levels. We verify the flow of negotiation through a simulation study.
\end{abstract}

\section{INTRODUCTION}

Cognitive radio networks can be modeled as autonomous agents acting in accordance with a set of conventions and regulations, typically embodied in policies. Radio physical link parameters are numerous and interrelated. Future cognitive radio networks are likely to be composed of heterogeneous software defined radios with highly diverse capabilities. Furthermore, they have differing missions and view of the environment. In order to conduct negotiations efficiently, the agent should have all the information that the other agents have. While one could envision a system which proactively ships knowledge bases around the network, this is inefficient as the environment continuously changes. Additionally, given many nodes in a region, only a few will need to connect to each other. Stated another way, information should be provided "just in time" in order to support the negotiations. This is the essence of argumentation based negotiations, where in addition to declaring an acceptance or rejection of a proposal, a rationale is provided. Proposals likewise include reasons why the recipient should accept.

In order to illustrate the capabilities of our approach, we examine a network composed of radio nodes communicating with a peer through a relay node. To establish a new connection, a radio node must negotiate with a relay node to handle its traffic. Each relay node has a maximum capacity, while each connection requires a particular rate and may have a priority status. Each radio has a limited and different knowledge of the system. They exchange ad evaluate proposals, possibly augmented with arguments, in accordance with a set of rules, in order to accomplish their communication goals in a constrained environment. This scenario allows us to consider a variety of topologies and initial conditions, in demonstrating the capabilities of the proposed protocolc to solve diverse problems.

This paper presents three contributions. We introduce argumentation based negotiation (ABN) in the context of cognitive radio networks (CRN), relating the theory of $\mathrm{ABN}$ to the practice of CRN. We also discuss how agents can create and evaluate proposals and arguments to negotiate over limited resources, under constraints. Finally, we describe our simulator which provides an implementation of this argumentation based negotiation system.

The rest of the paper is organized as follows. We next provide a brief overview of decision making in cognitive radio networks and review argumentation based negotiations in Section II. Three scenarios are then described in Section III to demonstrate feasibility and utility while providing a rich set of negotiating environments. Section IV presents our framework and how arguments are created and evaluated. Simulation environment, metrics and results are given in Section V. Finally, we provide a conclusion with some thoughts for future work in Section VI.

\section{RELATED WORK}

\section{A. Negotiation in Dynamic Spectrum Access Systems}

Negotiation in a broad sense occurs in many situations of a cognitive radio network practicing dynamic spectrum access. A number of researchers have applied various techniques to allow a cognitive engine in a radio agent to negotiate either with itself (essentially a self-reasoning process) or with other radio agents. Mitola has emphasized cognition at various levels of a radio's protocol stack, particularly at the application level where it interacts heavily with the user and his environment [1]. He also emphasizes case-based reasoning (CBR) as a means of synthesizing new solutions based upon past successes [2]. Buddhikot et al. considered auctions conducted by a centralized controller [3]. This system assumes that the controller has total knowledge of the 
environment. Rondeau and Rieser investigated using genetic algorithms in conjunction with CBR to find novel physical link layer parameter sets [4], [5]. This systems requires significant computing power and does not fully address the heterogeneity of capabilities and distributed knowledge. Neel et al. have discussed using game theory extensively to analyze cognitive radio networks and discover algorithms for achieving cooperative behavior [6], [7]. Game theory, in its original form, assumes that the parameters of the game are known by all participants. Considerable work is required to adapt this technology to systems with significant uncertainty and conflicting information amongst peers [8].

\section{B. Argumentation Based Negotiation}

Argumentation has roots in philosophy and can trace back to Aristotle [9]. In recent years, a number of researchers have begun to apply these concepts to artificial intelligence problems, first in self-reasoning applications and later in multi-agent systems [10]. Researchers in multi-agent systems often assume the agents are autonomous and rational [11]. Autonomous in this context means that the agent will make its own decision on whether to honor a request from another agent or whether it will initiate some action as a result of sensing something in its environment. Acting autonomously does not mean that the agent is not constrained by some set of rules, but that within these rules, it is free to decide on its course of action. Being rational means that it will act in its own interest [11].

Because one agent cannot force another agent to obey it, they are compelled to negotiate with each other in order to achieve their goals, when demand exceeds available resources. In fact, negotiation is even more important when one cannot guarantee that the agents will act rationally. This might occur if the agent's knowledge base is inconsistent or incorrect. Then the agent might make a decision which it thinks is in its interest; however, from an omniscient point of view, it is detrimental, and therefore, irrational.

As Rahwan et al. point out in their survey paper [8], "negotiation is needed when agents have competing claims on scare resources, not all of which can be simultaneously satisfied." They further assert that argumentation based negotiation can reach superior agreements faster by exchanging additional information about their beliefs and other mental attitudes in order to justify its own stance or influence another's stance. Specific types of information include a critique of another's statements (e.g. proposal), a justification of its own proposal, and information which changes the course of the negotiation process (e.g. introduce a relay node into a peer-peer link negotiation). Furthermore, threats or promises of a reward can be used to influence another's value system.

Dung [12] presents a logical view of argumentation via the concept of acceptability. If an agent cannot produce a counterargument which defeats an argument under consideration, then that argument under consideration must be accepted. Dung asserts "whether or not a rational agent believes in a statement depends on whether or not the argument support- ing this statement can be successfully defended against the counterarguments."

Sierra et al. focus on negotiation as persuading another agent to do something for it [13]. They emphasize the social roles that agents have with respect to one another. Their framework is dialogically focused and based upon the tuple $<$ Agents, Roles, R, L, ML,CL,Time>, where Roles is a set of social roles such as supervisor, subordinate, etc. that an agent could assume. $R$ is the mapping of agents to those roles. $L, M L$, and $C L$ represent the logical language, meta-language, and communication language respectively. The negotiations take place within a negotiation thread, with access to the history of negotiations. The thread begins with an offer or request dialog, followed by a series of proposals (including counter proposals) which may be rejected or accepted or one agent may withdraw from the negotiations. Each proposal message may include an offer, a threat, a reward, or an appeal. These are interpreted in the context of an authority graph generated from the roles of the respective agents. Amgoud and Vesic provide a formal analysis of the impact of exchanging arguments on the agents' theory and the added value of argumentation in general to negotiation systems [14]. They also demonstrate that argumentation can improve quality of an outcome, but never decrease it. Pasquier et al. extend argumentation to interest-based negotiation and provide a rare empirical insight into the actual implementation of an argumentation or interest-based negotiation system in software [15]. Finally, Mbarki et al. propose a constraintsbased argumentation system [16].

\section{SCENARIOS}

In order to illustrate the power of argumentation based negotiation in cognitive radio networks, we have created a set of scenarios where radio nodes negotiate for access with a certain grade of service in compliance with a set of rules. Each scenario consists of a set of radio nodes that communicate through relay nodes. They can communicate voice, standard video, or high definition video. Table I summarizes the rates assumed for each communication grade. There is an order to these in terms of the rate. Furthermore, each communication link can have either normal or high priority. Finally, there may be either one or two relay nodes.

TABLE I

COMMUNICATION GRADES AND RATES

\begin{tabular}{c|c|c}
\hline Grade & Rate & Unit \\
\hline \hline Voice & 256 & kbits/s \\
\hline Video & 3.5 & Mbits/s \\
\hline HDV & 8 & Mbits/s \\
\hline
\end{tabular}

The negotiation starts with a set of initial conditions composed of an existing communication link between nodes $N 1$ and $N 2$ as shown in Fig. 1, and a desire for $N X 1$ to communicate with $N X 2$, with a certain priority. Each radio agent's knowledge base includes specific and potentially different information about the topology, for example, the 


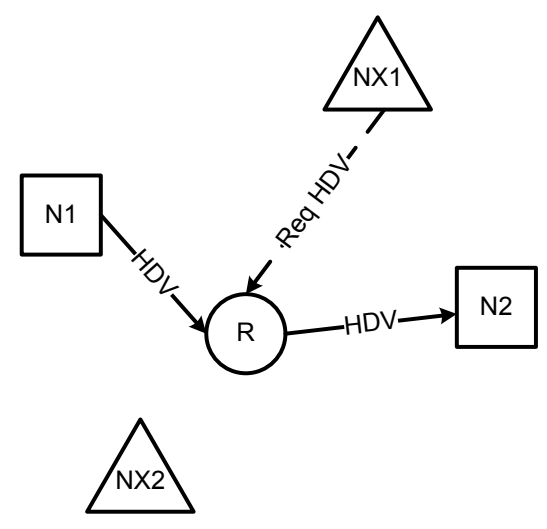

Fig. 1. Scenario 1 and 3 initial conditions.

existence of a second relay and the total capacity carrying capability of each relay node. The first step is for $N X 1$ to send a proposal to communicate to Relay1. Negotiations are resolved according to a specific set of rules, listed in Table II. Note that these are not the only set of rules one could compose for this problem; however, they are chosen to lead to reasonable results.

TABLE II

RULES FOR NEGOTIATION

\begin{tabular}{l|l} 
R1 & $\begin{array}{l}\text { All connections have a right to a class of } \\
\text { communications before any have a right to } \\
\text { a higher class(fairness). } \\
\text { All connections have a right to AUDIO } \\
\text { before any have a right to VIDEO or HDV. }\end{array}$ \\
R1.1 & $\begin{array}{l}\text { All connections have a right to VIDEO } \\
\text { before any have a right to HDV. }\end{array}$ \\
RIGH priority connections can force a \\
R2 & $\begin{array}{l}\text { NORMAL priority connection to down- } \\
\text { grade one level. }\end{array}$ \\
R3] & $\begin{array}{l}\text { HIGH priority connections cannot force a } \\
\text { disconnection of any existing connection. } \\
\text { Relays must accept handovers if it increases } \\
\text { the total number of connections in the net- } \\
\text { work. }\end{array}$
\end{tabular}

The first scenario starts with an ongoing HDV communication between $N 1$ and $N 2$ through relay $R$. The node $N X 1$ proposes an HDV connection to $N X 2$ through $R$. The agents follow a negotiation listed in Table III, and reach the end-state (see Figure Fig. 2). This scenario shows concession by $N X 1$ to drop to VIDEO in order to argue fairness (Rule R1.2), and a concession by $N 1$ when presented with that argument. It is implied that the relay node $R$ can support one HDV or two VIDEO connections, but not an HDV and VIDEO connection simultaneously.

The second scenario introduces a second relay, $R 2$, known to the first relay, $R 1$. The node $N X 1$ is aware of $R 1$, but not $R 2$. The relays negotiate a handoff as described in Table IV. The initial condition is given in Fig. 3, while the final condition is shown in Fig. 4.

The initial conditions for scenario three are the same as scenario one presented in Fig. 1. Here, the connection that $N X 1$ seeks to establish has a high priority. This negotiation
TABLE III

SCENARIO 1

\begin{tabular}{|c|c|}
\hline $\begin{array}{l}\text { Initial } \\
\text { Conditions }\end{array}$ & Existing HDV between N1 and N2 \\
\hline$\overline{\mathrm{NX} 1 \rightarrow \mathrm{R}}$ & Request HDV connection to NX2 \\
\hline $\mathrm{R} \rightarrow \mathrm{NX} 1$ & Denied. No capacity \\
\hline $\mathrm{NX} 1 \rightarrow \mathrm{R}$ & Request VIDEO connection to NX2 \\
\hline $\mathrm{R} \rightarrow \mathrm{NX} 1$ & Denied. No capacity \\
\hline $\mathrm{NX} 1 \rightarrow \mathrm{R}$ & Argue Rule R1 (fairness) \\
\hline $\mathrm{R} \rightarrow \mathrm{NX} 1$ & Agree Rule R1 applies \\
\hline $\mathrm{R} \rightarrow \mathrm{N} 1$ & Downgrade to VIDEO. Rule R1 applies \\
\hline $\mathrm{R} \rightarrow \mathrm{NX} 1$ & Request Granted. VIDEO to NX2 \\
\hline
\end{tabular}

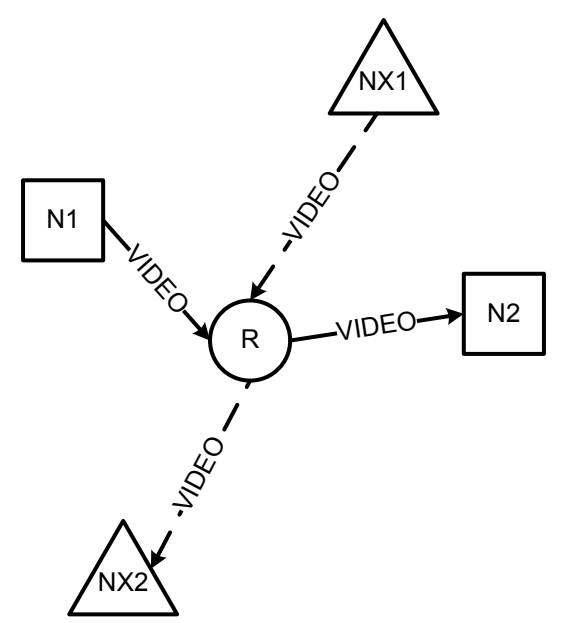

Fig. 2. Scenario 1 final conditions.
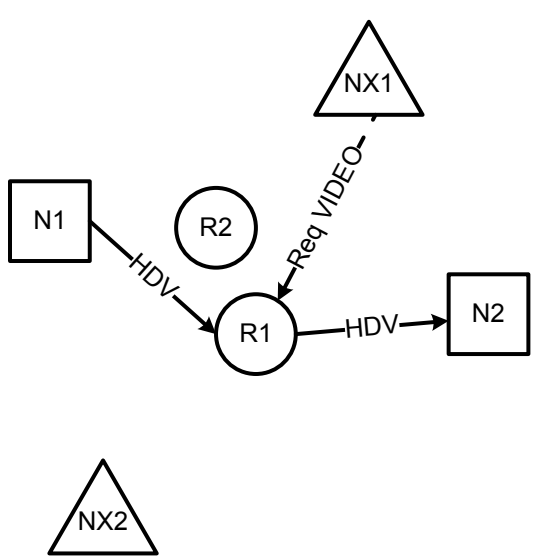

Fig. 3. Scenario 2 initial conditions.

TABLE IV

SCENARIO 2

\begin{tabular}{l|l}
$\begin{array}{l}\text { Initial } \\
\text { Conditions }\end{array}$ & $\begin{array}{l}\text { Existing HDV between N1 and N2 } \\
\text { Two Relay nodes }\end{array}$ \\
\hline \hline $\mathrm{NX} 1 \rightarrow \mathrm{R} 1$ & Request VIDEO to NX2 \\
$\mathrm{R} 1 \rightarrow \mathrm{NX} 1$ & Denied \\
$\mathrm{NX} 1 \rightarrow \mathrm{R} 1$ & Argue Rule R1 (fairness) \\
$\mathrm{R} 1 \rightarrow \mathrm{NX} 1$ & Agree Rule R1 \\
$\mathrm{R} 1 \rightarrow \mathrm{R} 2$ & HANDOFF request Connection \\
$\mathrm{R} 2 \rightarrow \mathrm{R} 1$ & N1-HDV-N2 \\
$\mathrm{R} 1 \rightarrow \mathrm{R} 2$ & HANDOFF denied \\
$\mathrm{R} 2 \rightarrow \mathrm{R} 1$ & Argue Rule 4 \\
$\mathrm{R} 1 \rightarrow \mathrm{NX} 1$ & Granted
\end{tabular}




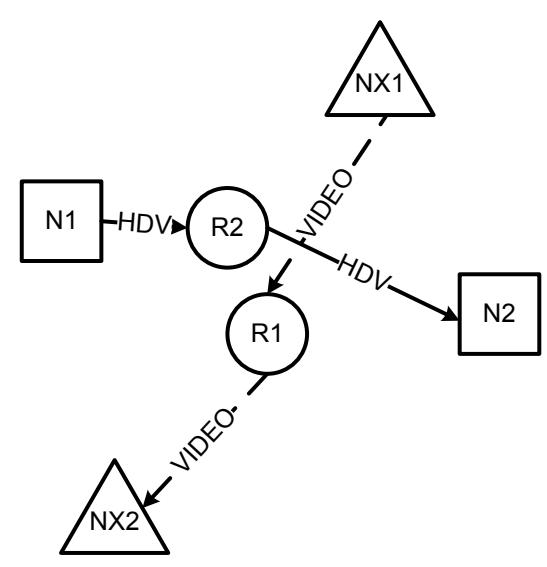

Fig. 4. Scenario 2 final conditions.

TABLE V

SCENARIO 3

\begin{tabular}{|c|c|}
\hline $\begin{array}{l}\text { Initial } \\
\text { Conditions }\end{array}$ & $\begin{array}{l}\text { Existing HDV between N1 and N2 } \\
\text { One Relay node. NX1 Priority }\end{array}$ \\
\hline $\mathrm{NX} 1 \rightarrow \mathrm{R}$ & Request HDV to NX2 \\
\hline $\mathrm{R} \rightarrow \mathrm{NX} 1$ & Denied \\
\hline $\mathrm{NX} 1 \rightarrow \mathrm{R}$ & Argue Rule R3 (Priority) \\
\hline $\mathrm{R} \rightarrow \mathrm{NX} 1$ & Agree Rule R3 \\
\hline $\mathrm{R} \rightarrow \mathrm{N} 1$ & Downgrade to VIDEO \\
\hline $\mathrm{N} 1 \rightarrow \mathrm{R}$ & Denied \\
\hline $\mathrm{R} \rightarrow \mathrm{N} 1$ & $\begin{array}{l}\text { Argue Rule R3. Node Request HDV } \\
\text { with Priority }\end{array}$ \\
\hline $\mathrm{N} 1 \rightarrow \mathrm{R}$ & $\begin{array}{l}\text { Accept downgrade to VIDEO. } \\
\text { Agree Rule R3 }\end{array}$ \\
\hline $\mathrm{R} \rightarrow \mathrm{NX} 1$ & Accept HDV to NX2 \\
\hline
\end{tabular}

follows Table V, with the end state given in Fig. 5. This negotiation forces the initial connection to concede when confronted with the priority argument. In this case, the relay node has a greater capacity than previous scenarios, being able to handle one HDV and one VIDEO, but not two HDV connections simultaneously. These scenarios provide a rich domain in which to study argumentation in negotiation for cognitive radio networks.

\section{FRAMEWORK}

\section{A. Negotiation Protocol}

A negotiation protocol describes what can be done at each step of the process. This may be to issue a proposal, accept a proposal, reject a proposal, amend a proposal, critique a proposal, offer a counter proposal, ask a question, or offer supplementary information. The philosophical study of argumentation in human dialog can be used to guide the development of a protocol [17]. This ultimately sets rules governing what statements can be uttered in a particular context and what the implications are of that utterance [18]. This analysis begins with the context describing the current state of the negotiation. For example, a context could be that another agent has submitted a proposal for consideration. One could imagine a number of legal responses in this context; however, in artificial intelligence applications, it is appropriate to consider constraining the set of legal responses. This not

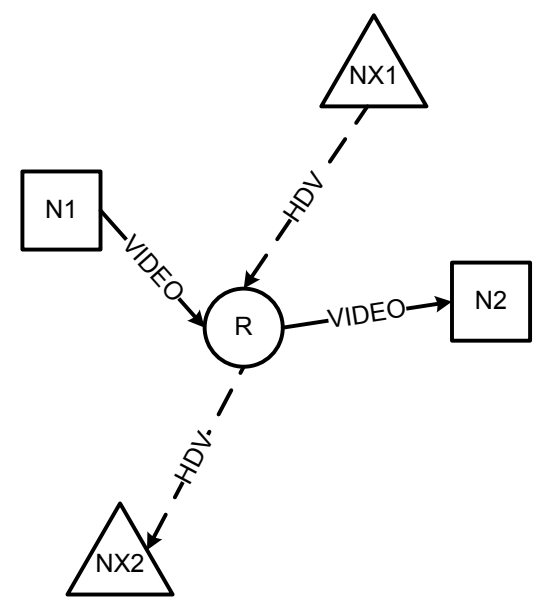

Fig. 5. Scenario 3 final conditions.

only simplifies the development effort, but also makes the system more predictable, potentially more stable, and likely faster. These issues are all critically important in our application.

Our intention is to use argumentation to speed the negotiation process and to find solutions that plain negotiation would overlook. Our first step is to develop an agent theory that will govern how agents select offers and arguments. Then we will develop a negotiating framework that describes how these offers and arguments may be exchanged.

Our framework assumes that any continuous parameters, such as rate, are discretized into a finite set of values. This means that the agent selects values rather than calculating a precise value. These selected values are formed into offers, the set of which is represented by $\mathcal{O}$. From the perspective of a radio node, there is a single negotiation with offers for a single communication link. On the other hand, from the perspective of a relay node, there are multiple simultaneous negotiations, each involving a communication link. The relay node is concerned with a conjunction of offers and commitments. For example, in scenario 1, it initially considers the conjunction of the commitment of the ongoing communication between $N 1$ and $N 2$ and the proposal for communication between $N X 1$ and $N X 2$. Ultimately, it opens a negotiation with $N 1$ to downgrade its link with $N 2$ and considers this negotiation in concert with the proposal from $N X 1$.

TABLE VI

POSSIBLE OFFERS FROM NX1 WITH TWO RELAY NODES

Offer $<N X 1, N X 2, R 1, H D V>$
Offer $<N X 1, N X 2, R 1, V I D E O>$
Offer $<N X 1, N X 2, R 1, V O I C E>$
Offer $<N X 1, N X 2, R 2, H D V>$
Offer $<N X 1, N X 2, R 2, V I D E O>$
Offer $<N X 1, N X 2, R 2, V O I C E>$

According to [14], arguments can be classified as epistemic or practical. Epistemic arguments arise from and justify beliefs. Practical arguments justify offers and are built from both beliefs and goals. In scenario 2, the HIGH priority of the link 
between $N X 1$ and $N X 2$ is a fact that can form an epistemic argument. An example of a practical argument is the desire or goal of $N X 1$ to communicate with $N X 2$. Epistemic arguments are represented by $\operatorname{Arg}_{e}(\mathcal{L})$. and practical arguments by $\operatorname{Arg}_{p}(\mathcal{L})$. Some examples are listed in Table VII.

TABLE VII

POSSIBLE PRACTICAL ARGUMENTS OF NX1

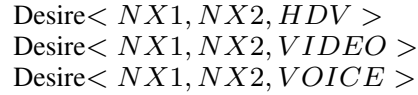

Table VIII lists several facts that can be used to generate an epistemic argument. The first line could be used to argue for the video link between $N X 1$ and $N X 2$ to be granted due to its high priority status. The second and third lines could form an argument to convince Relay 2 to accept a hand off. The last line could be used by $N X 1$ to argue that it should be granted a VID link to $N X 2$ by asserting Rule 1.2.

TABLE VIII

FACTS LEADING TO POSSIBLE EPISTEMIC ARGUMENTS

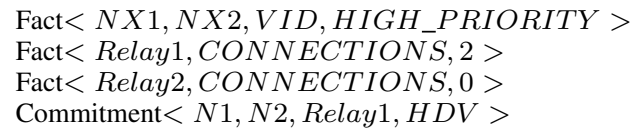

Each exchange in a negotiation is a message that may be a proposal, an argument, acceptance or rejection of a proposal, agreement or disagreement with an argument. Proposals and responses can be augmented with an argument, although arguments can be sent on their own. This system implies that participating nodes keep state about the negotiation process.

\section{B. Argument Generation and Evaluation}

For each argument, we consider when it applies, how to evaluate it for acceptance or rejection, and how to respond for each result. We also consider how the issuing agent should respond if the receiving agent accepts or rejects the argument. Our arguments are narrowly defined in the context of our carefully designed scenarios. This allows us to reasonably bound the conditions for generation and evaluation. We analyze each argument individually, starting with the fairness argument.

\section{Fairness}

Applies Assert when a proposal has been rejected after asking for less than the highest rate handled by the relay. The requesting node will typically not know the highest rate currently handled by the relay, but will know the highest rate defined in the negotiating system. In this case, that is HDV. This argument cannot be asserted when requesting $\mathrm{HDV}$, but it can when requesting VIDEO or VOICE.

Evaluation The receiving agent should agree with this argument if it can find at least one connection with normal priority at a rate greater than the one requested.

Acceptance The receiving agent should reevaluate the associated proposal to see if there is a connection that can be downgraded and issue a proposal accordingly.

Rejection The receiving agent should send a DISAGREE message to the originating agent, which will in turn attempt to find another argument to support the proposal, or issue a new proposal with a concession.

\section{Priority}

Applies Assert when rejected, if the requesting node has priority.

Evaluation Accept if there is a higher or equal rate connection without priority.

Acceptance Create DOWNGRADE proposal with a priority argument and send to a node of the existing connection without priority

Rejection Send DISAGREE message to originator. The originating node may concede and issue a proposal at a lower rate, and subsequently argue for fairness.

\section{Favor Increase in Connections}

Applies Assert by a relay node when another relay node has rejected a HANDOFF proposal

Evaluation Accept if it can support the HANDOFF without having to drop an existing connection

Acceptance Reevaluate the original proposal that triggered the HANDOFF proposal and accept it if possible.

Rejection Send a REJECT notice to the node that originated the proposal that triggered the HANDOFF proposal (if no other relays are available). The originating node may attempt a new argument, such as priority, or concede and submit a proposal at a lower rate.

\section{Performance of ABN Framework}

The key metrics in negotiation are the speed at which a deal can be met, the quality of the deal, the robustness in proceeding to a deal, and the ability to conclude negotiations even when a deal cannot be met. From our perspective, optimality of the deal is preferred; however, not necessarily at the cost of stability and speed. This is a function of the application domain. A negotiating system governing spectrum allocation of femto cells in a 4G network might favor spectrum efficiency over speed of negotiation, since they operate in a relatively static environment. A DSA system operating in the public safety band encounters a much more dynamic environment, thereby favoring speed of negotiation over optimum spectral efficiency. Although Amgoud has made progress towards demonstrating that $\mathrm{ABN}$ can reach superior deals [14], optimality is not yet within reach. 


\section{Simulation Study}

\section{A. Simulation envrionment and metrics}

We created a Java based simulation program which accepts a set of initial conditions and negotiates a solution according to the set of rules adopted. Proposals, arguments, and responses are exchanged by messages directed from an originator to a target. A proposal for a connection includes a source, destination, communication grade, and relay. Note that the originator and target do not necessarily correspond to a source or destination. In fact, a radio node acting as a source will typically originate a proposal targeted to the relay it wants to handle its traffic.

Both relays and basic radio nodes inherit from a common object, ABNAgent, that supports negotiations. Each negotiating agent maintains a knowledge base of facts that originate from either the initial conditions or arguments. These facts include the existence of different entities, such as Relay2, and connection priority. A list of existing connections is maintained so that agents can calculate their current load and find existing connections to modify (e.g. handoff or downgrade) in order to support a new proposal. Finally, a list of pending proposals is managed as agents issue new proposals in response to received or rejected proposals. This mechanism allows a relay node, upon receiving a request for a new connection that exceeds its capacity, to negotiate with a node in an ongoing communication to modify its connections, and ultimately respond to the original requesting node, based upon the outcome of the secondary negotiation.

A negotiating agent moves through several different states during negotiations, as illustrated in Fig. 6. Negotiation starts when an agent is seeded with a desire, such as communicate HDV with NX2. This desire becomes an intention as it creates a proposal to a relay entity, found by examining its knowledge base. The node then enters a waiting state until it receives a response.

Valid responses from the relay node can be acceptance or rejection of the proposal. The originating node then attempts to create an argument supporting the original proposal. If it can find one, it sends it; otherwise, it attempts to create a new proposal. For example, if it cannot find an argument to support an HDV connection, it may create a new proposal to create a VIDEO connection. Each argument can only be sent once. When the arguments supporting a proposal are exhausted, then a concession is required in order to submit a new proposal to the same agent. When the originating node is unable to generate a new argument or proposal, it reaches a "no deal" conclusion.

Agents evaluate proposals by calculating the new load and comparing that to the agent's maximum capacity. If they can support the new connection in addition to their current connections, the agent will accept the proposal. The exception to this is a HANDOFF request to a relay node. In this case, the default is to refuse unless there is a supporting argument. This seems reasonable since a handoff to a different relay carries with it some risk of a dropped connection if the end nodes

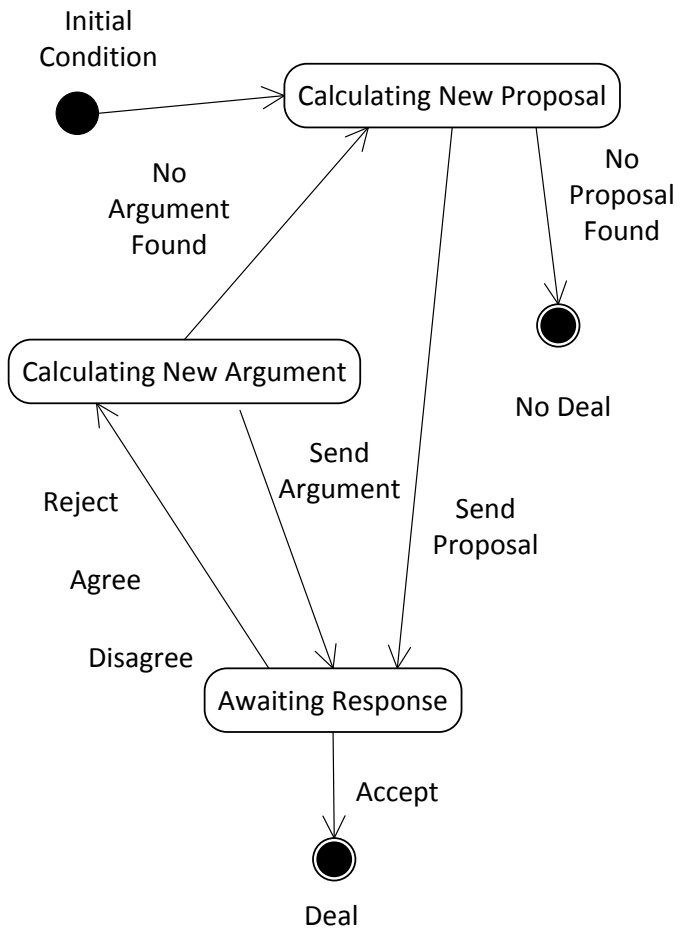

Fig. 6. Radio node negotiation state diagram.

cannot reach it.

In general, nodes issue their strongest arguments first; however, it is not always possible to ascertain the most effective argument. Knowledge of another relay node can be more effective than asserting priority, unless that other relay node is already carrying priority traffic at its maximum capacity.

We are able to seed the simulation with various initial conditions, observe the negotiation process and examine the resulting deal. The simulation starts with an existing connection and negotiates a new connection, as in the scenarios described earlier. The various initial conditions are the capacity of the relay nodes, the grade of the existing connection, the priority condition of both the existing connection and the proposed connection, the existence of a second relay node, and the desired grade of the new connection. A large subset of the possible trials are presented in Table IX. In all cases, the capacity of the relay nodes is $12 \mathrm{M}$, so that each relay can support either one HDV and one VIDEO connection or two VIDEO connections. The inability to support two HDV connections through a single relay creates a resource constrained environment, necessitating negotiations. We also present some trials where the capacity is not exceeded by the additional connections, to demonstrate the stability of the system to all types of conditions.

\section{B. Simulation results}

In reviewing the resulting solutions for a wide variety of initial conditions in Table IX, we observe that the argumentation based negotiation leads to reasonable results. This is 
TABLE IX

SIMULATION RESULTS

\begin{tabular}{r|c|c|c|c|c|c|c|c}
\hline & \multicolumn{4}{|c|}{ Existing } & \multicolumn{3}{c|}{ Desired } & \multicolumn{3}{c}{ Solution } \\
Trial & Service Class & Priority & Relays & Service Class & Priority & Prior Service & New Service & Num Messages \\
\hline 1 & HDV & NONE & 1 & HDV & NONE & HDV & VID & 4 \\
\hline 2 & HDV & NONE & 1 & HDV & HIGH & VID & HDV & 7 \\
\hline 3 & HDV & NONE & 2 & HDV & NONE & HDV & HDV & 3 \\
\hline 4 & HDV & HIGH & 1 & HDV & NONE & HDV & VID & 4 \\
\hline 5 & HDV & HIGH & 1 & HDV & HIGH & HDV & VID & 10 \\
\hline 6 & HDV & HIGH & 2 & HDV & HIGH & HDV & HDV & 3 \\
\hline 7 & VID & NONE & 1 & HDV & NONE & VID & HDV & 2 \\
\hline 8 & VID & NONE & 2 & HDV & NONE & VID & HDV & 2 \\
\hline 9 & VID & HIGH & 1 & HDV & NONE & VID & HDV & 2 \\
\hline
\end{tabular}

noteworthy since the design of the rules and negotiation system were based upon three scenarios, yet when many different combinations of the initial conditions are considered, the system still behaves well.

An often cited advantage of argumentation systems is the ability to find solutions that basic negotiations cannot. We see this in particular when introducing a second relay node. The originating node, $N X 1$, does not know of the existence of the second relay node; however, the first relay node does have this knowledge, and acts accordingly to find a solution where no concessions are required (other than a handoff). Another situation arises when both the existing and proposed connection have a high priority and are requesting more aggregate resources than the relay can provide. This system results in an orderly conclusion, although a concession is required.

The simulation and scenarios are setup to send proposals and arguments separately. This helps to expose more detail about the negotiating process; however, one could also design the system to send the strongest argument available, whenever sending a proposal. While this would reduce the communication overhead in an implemented system, it has no significant impact on the negotiation process, except in those cases where a proposal is accepted without regard to any argument. These are trivial conditions where negotiation is not required. None of the trials listed in Table IX resulted in a "No Deal", because the initiating agent preferred a lower rate to no rate at all. In other words, if rejected when requesting HDV service and no arguments prevailed, then $N X 1$ will ask for VIDEO service instead. In case this is not acceptable, a minimum communication grade fact can be added to $N X 1$ 's knowledge base. The NewProposal algorithm will check that before concession and conclude that no proposal can be generated, resulting in the "No Deal" termination condition.

Although a "No Deal" termination seems like a failure, it is an acceptable response. It is crucial that the negotiations terminate when a solution is not available, in order to minimize futile resource consumption. The system can handle a "No Deal" response by queuing the request, or take other action as dictated by the higher level application.

The arguments in the simulation supporting these scenarios are assertions, but in some situations, there is an implied inquiry. Sending a high priority argument is an assertion.
Sending a fairness argument is an assertion, with an implied inquiry. In our scenarios, the proposing node does not know the communication grades of the other connections that the relay is carrying. If the relay accepts the proposal (after internally agreeing to the argument), the proposing node can conclude that there had been a connection at a higher rate and that it did not have a high priority. If the relay agrees to the argument, but is still unable to accept the proposal, then the proposing node can infer that the relay is carrying a large number of minimal grade (AUDIO) connections with a limited maximum capacity, and is unable to find capacity due to rule R3. If the relay disagrees with the argument, then it implies that all other connections have priority asserted also. Rather than issuing an inquiry first, and then a fairness argument, it is more efficient in many cases to directly issue the fairness argument.

Furthermore, there is an implied trust, in that arguments are accepted if they cannot be defeated, consistent with Dung's acceptability criteria [12]. This is illustrated by the relay asserting the fairness argument towards N1. This node accepts the argument because it cannot defeat it. An interesting attack relationship is found when both the initial and the proposed connections have a high priority. Each priority assertion attacks the other, resulting in a stalemate. With no other options, such as a second relay, the proposing node is forced to concede to VIDEO grade as a de facto first-come-first-served rule applies.

Rogue radio nodes could simply assert that they have a High Priority status, even when they do not. One way of dealing with this is to require the node to send a certificate traceable to an authority that grants the priority status. Network admission control techniques can also be deployed to ensure that only well behaving nodes are allowed entry into the negotiating process. Finally, the argumentation system can be extended to demand that the radio node present arguments supporting its High Priority status assertion. A typical argument might state that the information is of a tactical nature, where the success of a mission and preservation of lives is at stake. While this may allow the relay node to arbitrate with a finer grained resolution between potentially conflicting priority assertions, some secure authentication mechanism will eventually be needed to ensure that the arguments are based in truth. The level of secure authentication is application dependent. 
Overhead is a concern in capacity constrained networks. We introduce overhead by the act of negotiating and further add to it when exchanging arguments. Of course, much research is directed at finding solutions without needing to incur the overhead of negotiation. Nevertheless, one can easily conceive of circumstances where the nodes of a system are heterogeneous (in terms of capability) and the local information at each node is incomplete or even inconsistent with other nodes. Information exchange can clearly lead to superior configurations relative to solutions without any information exchange. Argumentation actually improves the overall overhead by providing information to guide a negotiation, rather than letting the negotiation iterate throughout a search region. In a sense, it is the tool to rapidly and directly move from a game of imperfect information to one of perfect information.

The actual calculation of overhead is dependent upon application and domain issues such as the length of the aforementioned certificate authenticating the High Priority argument. It is also a function of whether or not anticipatory arguments are sent with an original proposal, or in response to a rejection. For the scenarios we have described, the strongest argument, or even all arguments, could always be sent to advantage. As systems scale with complexity of rules and arguments it will become more important to choose the best (i.e. strongest or most relevant) argument. By sending only the strongest argument, the task of evaluating the arguments is eased (at the expense of more difficulty in choosing the best one). The number of message exchanged column in Table IX provides a qualitative examination of the overhead required. These values include the original proposal and the accept message.

\section{CONCLUSION}

We have shown that argumentation based negotiation in the context of a set of rules leads to reasonable outcomes for a broad set of initial conditions. It is also able to find solutions that are not apparent to the initial proposer in the negotiation. The argumentation system effects an exchange of information, but properly preserves conflict relationships. Finally, by designing the argumentation system around three scenarios, we demonstrated that the system generalizes reasonably when presented with other scenarios.

This simple set of scenarios provide a rich analysis of the feasibility and utility of argumentation based negotiation in cognitive radio networks. There are many situations where distributed decision making over constrained resources with conflicting information complicates conventional decision processes. Argumentation based negotiation can be applied to many of these problems. It will be particularly useful in dynamic problems where there is a peer relationship, or illdefined authoritative structure, amongst heterogeneous nodes. Argumentation can replace static authority models, facilitate resolution of conflicting data, and cope with different node capabilities.

\section{REFERENCES}

[1] J. Mitola III, Cognitive Radio Architecture: The Engineering Foundations of Radio XML. Wiley-Interscience, 2006.

[2] — "Cognitive radio an integrated agent architecture for software defined radio," Ph.D. dissertation, Royal Institute of Technology, 2000

[3] M. Buddhikot, P. Kolodzy, S. Miller, K. Ryan, and J. Evans, "Dimsumnet: New directions in wireless networking using coordinated dynamic spectrum access," in IEEE International Symposium on a World of Wireless, Mobile and Multimedia Networks, June 2005, pp. 78-85.

[4] T. W. Rondeau, C. J. Rieser, B. Le, and C. W. Bostian, "Cognitive radios with genetic algorithms: Intelligent control of software defined radios," in SDR Forum Technical Conference, 2004.

[5] C. J. Rieser, "Biologically inspired cognitive radio engine model utilizing distributed genetic algorithms for secure and robust wireless communications and networking," Ph.D. dissertation, Virginia Polytechnic Institute and State University, 2004.

[6] J. Neel, R. Buehrer, B. Reed, and R. Gilles, "Game theoretic analysis of a network of cognitive radios," in 45th Midwest Symposium on Circuits and Systems MWSCAS, August 2002, pp. 409-412.

[7] J. Neel, J. H. Reed, and R. P. Gilles, "The role of game theory in the analysis of software radio networks," in SDR Forum Technical Conference, 2002.

[8] I. Rahwan, S. D. Ramchurn, N. R. Jennings, P. McBurney, S. Parsons, and L. Sonenberg, "Argumentation-based negotiation," in The Knowledge Engineering Review. Cambridge University Press, Dec 2003, vol. 18, pp. 343-375.

[9] Aristotle, Topics, W. D. Ross, Ed. Oxford, UK: Clarendon Press, 1928, (Original work published c. 350 BCE).

[10] K. Sycara, "Persuasive argumentation in negotiation," Theory and Decision, vol. 28, pp. 203-242, 1990.

[11] M. Wooldridge, An Introduction to Multiagent Systems. John Wiley \& Sons, Ltd, 2002.

[12] P. M. Dung, "On the acceptability of arguments and its fundamental role in nonmonotonic reasoning, logic programming and -person games," Aritificial Intelligence, vol. 77, no. 2, pp. 321-357, September 1995.

[13] C. Sierra, N. R. Jennings, P. Noriega, and S. Parsons, "A framework for argumentation-based negotiation," in Intelligent Agents IV: Agent Theories, Architectures, and Languages: 4th, ser. Lecture Notes in Artificial Intelligence, M. P. Singh, A. Rao, and M. J. Wooldridge, Eds., vol. 1365. Springer-Verlag, July 1997, pp. 117-192.

[14] L. Amgoud and S. Vesic, "A formal analysis of the role of argumentation in negotiation dialogues," Journal of Logic and Computation, 2011.

[15] P. Pasquier, R. Hollonds, I. Rahwan, F. Dignum, and L. Sonenberg, "An empirical study of interest-based negotiation," Autonomous Agents and Multi-Agent Systems, vol. 22, no. 2, pp. 249-288, 2011.

[16] M. Mbarki, J. Bentahar, B. Moulin, and A. Moazin, "Constraintsbased negotiation using argumentation," in Proceedings of the 13th International Workshop on Non-Monotonic Reasoning (NMR), 2010.

[17] D. N. Walton and E. C. W. Krabbe, Commitment in Dialogue: Basic Concepts of Interpersonal Reasoning. State University of New York Press, 1995

[18] L. Amgoud, S. Parsons, and N. Maudet, "Arguments, dialogue, and negotiation," in Proceedings of the 14th European Conference on Artificial Intelligence, W. Horn, Ed. Amsterdam: IOS Press, 2000. 